\title{
Static Friction in Elastic Adhesive MEMS Contacts, Models and Experiment
}

\author{
N.R. Tas, C. Gui, and M. Elwenspoek \\ MESA Research Institute, University of Twente \\ P.O. Box 217, 7500 AE Enschede, the Netherlands \\ e-mail: N.R.Tas@el.utwente.nl
}

\begin{abstract}
Static friction in shearing mode can be expressed as the product of the shear strength of the interface and the real contact area. The influence of roughness on friction in elastic adhesive contact is analyzed. Special attention is paid to low loading conditions, in which the number of contact points is small. The models are used to analyze a friction experiment in a MEMS friction meter.
\end{abstract}

Keywords: Static Friction, adhesion, contact mechanics

\section{INTRODUCTION}

\subsection{General}

The application of friction reducing elements, like ball bearings, magnetic levitation and hydrostatic bearings is often too complicated in MEMS devices. Therefore, dry sliding friction occurs in most bearings and joints. Tests of the first electrostatic micromotors showed a significant effect of friction on micromotor performance $[1,2]$. Therefore, in situ static friction measurements have been done, yielding friction coefficients up to $4.9 \pm$ 1 [3]. Due to the large surface to volume ratio in MEMS devices, adhesion forces become relatively large, and in some cases lead to a friction force much larger than the applied normal force $[2,4,5]$. Measurement, reduction and control of friction have become a major concern in the development of micromechanisms [5-15]. One of the striking outcomes of these studies is the wide range of the measured static friction coefficients for the structural materials used in MEMS [16]. Differences in the surface topography can be one of the causes of the spread in the measured friction coefficient. Therefore, the static friction dependence on surface topography will be analyzed in this paper. In MEMS devices the typical contact materials are silicon, silicon dioxide and silicon nitride, which are rather hard. Therefore, in most contacts elastic deformation occurs, and elastic contact models $[17,18]$ can be used. Because the aim of this paper is to explain the basic physics of static friction, we prefer to use these models with a simple surface topography representation. An overview of more refined representations is given by Majumdar et. al. [19]. In MEMS devices a wide range of surface roughness is found, varying from the almost atomically smooth surface of a chemical-mechanically polished silicon wafer, to the rough surface of deposited polysilicon.
From stiction and wafer bonding experiments it is known that the adhesive attractive forces between contacting surfaces drop rapidly with increasing surface roughness $[20,21]$. However, the load forces in MEMS devices are often so small ( $\mu \mathrm{N}$ 's), that even in rough contacts the contribution of the adhesion forces to the total load compressing the surfaces can not be neglected [22]. The influence of adhesion, and its dependence on the surface roughness is therefore included in the elastic contact model.

\subsection{Static Friction Modelling}

Following Bowden and Tabor [23], two basic friction mechanisms can be distinguished in sliding contacts: ploughing of the asperities of the harder material through the softer material, and shearing of the junctions formed in the region of contact. In this paper we focus on the shearing friction, because the interfaces in MEMS are often between almost equally hard materials, and the asperities are mostly rather blunt. Bowden and Tabor [23] give the shearing friction force by:

$$
F_{\mathrm{f}}=s \cdot A_{\mathrm{r}}
$$

Where $s$ is the shear strength of the interface and $A_{\mathrm{r}}$ is the real area of contact. The real contact area is given by $A_{\mathrm{r}}=L / p_{\mathrm{m}}$ with $L$ the (externally) applied load and $p_{\mathrm{m}}$ the mean real contact pressure. In case the asperities deform plastically the friction force is given by $F_{\mathrm{f}}=$ $\mu_{\mathrm{p}} \cdot L$, with $\mu_{\mathrm{p}}=s / H$ and $H$ the hardness of the contacting materials [24]. In case the asperities touching the counter surface are spherical and deform elastically, the contact area for each asperity is given by the Hertz's theory. If all asperities were located at the same height, the contact area would increase with $L^{2 / 3}$. For real surfaces and many contact points, the asperity summit level is distributed, and the area of contact becomes proportional to $L$ [17]. The friction force is now given by $F_{\mathrm{f}}=\mu_{\mathrm{e}} \cdot L$ with $\mu_{\mathrm{e}}=s / p_{\mathrm{c}}$ and $p_{\mathrm{e}}$ the mean real elastic contact pressure. So, for both elastic and plastic contact the friction as a function of the applied load fulfills Amontons' Law:

$$
F_{\mathrm{f}}=\mu \cdot L
$$

Because $\mu=s / p_{\mathrm{m}}$ it is easy to understand the lowering of the friction coefficient by low adhesive monolayer coatings [25-29]: They reduce the shear strength of the 
interface, while due to the small thickness of the coating the elastic properties of the interface are not altered. For elastic contact, the real contact area and thus the contact pressure can be calculated using elastic contact mechanics models $[17,18]$. These models will therefore be explained next. First, the elastic deformation of a single asperity as given by Hertz' theory is introduced. Extensions to include the effect of adhesive load are made. In section 3.1 we explain the Greenwood and Williamson theory [17]. It relates the contact force, the contact area, the number of contact points, the surface topography and the elastic properties of the contacting surfaces, for multi-asperity contacts. Maugis extension [18] to include the effect of adhesive load is given in section 3.2. These contact models can be used to derive expressions for the friction force, and make it possible to explain the empirical Amontons' Law. The influence of surface topography, adhesion energy, elastic and plastic properties of the contacting surfaces on the friction coefficient will be analyzed. We have found that for low loading conditions the number of contacting asperities can be so small that the statistical models can not be used. In particular, the influence of the adhesive load is completely miscalculated. For low loading conditions we therefore present a model which assumes a minimum number of contact points, as in contact there should always be at least one contact point (section 4). Finally, in section 5 we apply the friction theory to a friction experiment in a MEMS device.

\section{SINGLE ASPERITY CONTACT}

For one spherical asperity deforming elastically, the relation between the contact radius $a$, the indentation $z$ and the compressive force $P$ pressing the asperity to a flat surface is given by Hertz's equations [30, 31]:

$$
\begin{aligned}
& a^{2}=R \cdot z \\
& P=\frac{a^{3}}{R \cdot D}=\frac{R^{1 / 2} z^{3 / 2}}{D}
\end{aligned}
$$

Where $D$ is the composite compliance of the two contacting materials, defined as:

$$
D=\frac{3}{4} \cdot\left(\frac{1-v_{1}^{2}}{E_{1}}+\frac{1-v_{2}^{2}}{E_{2}}\right)
$$

Where $E_{1,2}$ and $v_{1,2}$ are the Young's modulus and the Poisson ratio of the two surfaces respectively. To include the effect of adhesion, different single asperity models have been proposed. For reasons of simplicity we use the DMT model [32], which assumes that the elastic deformation of a contacting asperity is described by Hertz's equations, also if there are adhesion forces pulling the asperity and the counter surface together. The adhesion forces are simply added to the load, and the expressions for the contact radius $a$ and the load $P$ as a function of the indentation become:

$$
\begin{aligned}
& a^{2}=R \cdot z \\
& P+2 \pi \cdot \Delta \gamma \cdot R=\frac{a^{3}}{R \cdot D}=\frac{R^{1 / 2} z^{3 / 2}}{D}
\end{aligned}
$$

With $\Delta \gamma\left[\mathrm{J} / \mathrm{m}^{2}\right]$ the work of adhesion. In case that the deformation profile is changed due to adhesion forces acting around the perimeter of the contact circle (formation of a 'neck'), one should consider to use the JKR model [33]. Which model to use, the DMT or the JKR model, depends on Tabor's elasticity parameter [34].

\section{MULTI ASPERITY CONTACT}

\subsection{No adhesive load: The Greenwood and Williamsen model}

Greenwood and Williamson [17] have modeled the elastic contact of nominally flat and parallel surfaces, of which one is smooth and the other is rough. Adhesion is neglected in the model. The surface roughness is represented by asperities, which at least near their summits, are spherical with a radius $R$. For two rough surfaces the composite $R=\left(1 / R_{1}+1 / R_{2}\right)^{-1}$ is used. The other parameters describing the surfaces are the elasticity of the materials, the asperity density $\eta\left[\mathrm{m}^{-2}\right]$, and the spread $\sigma$ in the asperity summit levels. For two rough surfaces $\eta=\eta_{1}+\eta_{2}$. The asperities have a summit level distribution $\phi(x)$ where $\phi(x) \mathrm{d} x$ is the probability to find an asperity with a summit level between $x$ and $x+\mathrm{d} x$ above a reference plane in the rough surface (see fig. 1). The level of the smooth plane is $d$ above the reference plane at $x=0$, and $d$ is called the separation.

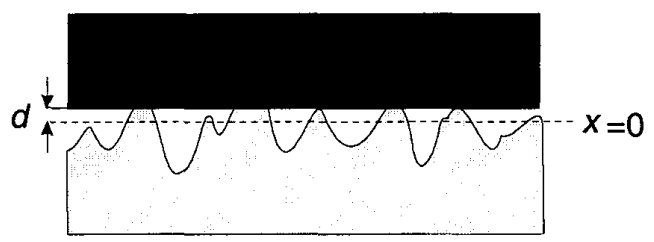

Figure 1: The separation $d$ is defined as the level of the smooth plane with respect to the reference plane at $x=0$.

The asperity height distribution depends on the surface preparation prior to the contact. In engineering practice the summit level distribution is often close to Gaussian [17]. It is therefore assumed here that the distribution is Gaussian with a standard deviation $\sigma$ and centered around $x=0$. For two rough surfaces the composite standard deviation $\sigma^{2}=\sigma_{1}^{2}+\sigma_{2}^{2}$ is applied. The asperity heights $x$ and the separation $d$ can be normalized according to $y=x / \sigma$ and $h=d / \sigma$. The expressions for the number of contact points $N$, the real contact area $A_{\mathrm{r}}$ and the load $L$ have been derived by Greenwood and Williamson [17]. In the dimensionless representation [18] the relations become: 


$$
\begin{aligned}
& N^{*}=\frac{N}{\eta A_{a}}=F_{0}(h) \\
& A^{*}=\frac{A_{r}}{\eta R \sigma A_{a}}=\pi F_{1}(h) \\
& L^{*}=\frac{D \cdot L}{\eta A_{a} R^{1 / 2} \sigma^{3 / 2}}=F_{3 / 2}(h)
\end{aligned}
$$

For the Gaussian distribution of the asperity summit levels the normalized probability integrals are given by:

$$
F_{n}(h)=\frac{1}{\sqrt{2 \pi}} \int_{h}^{\infty}(y-h)^{n} e^{-\frac{y^{2}}{2}} d y
$$

The integrals in eq. $(5 \mathrm{a}, \mathrm{b}, \mathrm{c})$ are taken to add the contributions of all asperities in contact. The dimensionless group $\eta R \sigma$ in eq. ( $5 \mathrm{~b}$ ) has been shown to be in the range of $0.05-0.1$ by Archard [35]. It is characteristic for the Gaussian distribution, that the normalized contact pressure $L^{*} / A^{*}$ as a function of $L^{*}$ is almost constant. It varies less than a factor 2 over three decades of $L^{*}$. The explanation for this is that as the load increases, the number of contact points increase almost proportional (power $\sim 0.9$ ). The real mean contact pressure and the real contact area per asperity therefore remain almost constant. The load independence of the $p_{\mathrm{e}}$ is important in relation to eq. (1). It largely eliminates the load dependence of $s$ in case $s$ is pressure dependent. The expression for the mean real elastic contact pressure $L / A_{\mathrm{r}}$ can be found by combination of eq. $(5 b, 5 c)$.

$$
p_{e}=\frac{L}{A_{r}}=\sqrt{\frac{\sigma}{R}} \cdot \frac{F_{3 / 2}(h)}{D \pi F_{1}(h)}
$$

In section $3.3, p_{\mathrm{e}}$ is used to analyze the effect of the surface topography on the friction coefficient.

\subsection{Including adhesive load}

To include adhesion, the statistical integrals are calculated using the DMT model (eq.(4)). The expressions for $A_{\mathrm{r}}, N, A^{*}, N^{*}$ remain the same. Eq. (5c) changes into:

$$
L^{*}=\frac{D \cdot L}{\eta A_{a} R^{1 / 2} \sigma^{3 / 2}}=F_{3 / 2}(h)-\frac{2 \pi}{\theta} F_{0}(h)
$$

In which $\theta$ is the adhesion parameter, defined by Fuller and Tabor [36]:

$$
\theta=\frac{\sigma^{3 / 2} R^{1 / 2}}{D \cdot \Delta \gamma \cdot R}
$$

The adhesive load is proportional to the number of contacting asperities. It is extremely sensitive to changes in the surface roughness. This can be shown by calculation of fraction of contacting asperities as a function of the adhesion parameter, using eq. (5a) and (8): For $L^{*}=0$ and $\theta$ increasing from 1 to 30 the fraction of contacting asperities decreases from 1 to 0.001 (fig. 2). At the same time the relative real contact area $A_{\mathrm{r}} / A_{\mathrm{a}}$ decreases from 1 to 0.0001 , assuming $\eta \cdot R \cdot \sigma$ $=0.1[21]$.

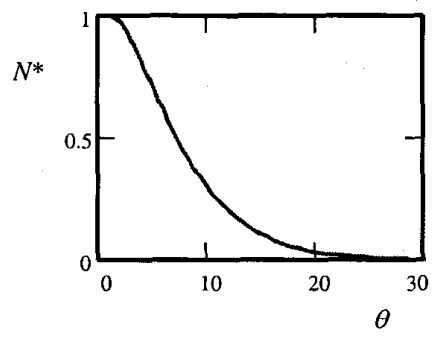

Figure 2: The fraction of asperities in contact as a function of the adhesion parameter, for $L^{*}=0$.

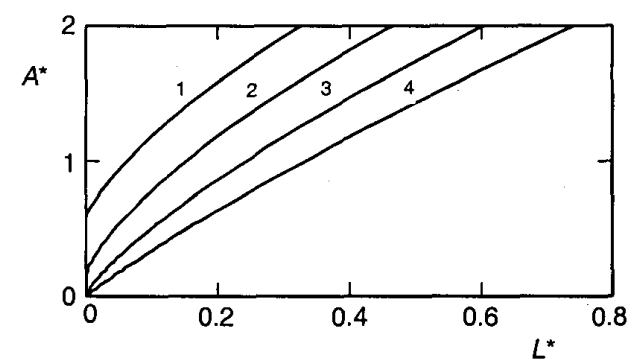

Figure 3: $A^{*}$ vs. $L^{*}$ for (1) $\theta=10$, (2) $\theta=15$, (3) $\theta=30$ and (4) $\theta=\infty$.

Using eq. $(5 \mathrm{~b}, 8)$ the normalized contact area $A^{*}$ as a function of the normalized applied load $L^{*}$ can easily be plotted for four different values of $\theta$, (fig. 3). Based on eq. (1) the static friction as a function of the applied load will have this shape. Fig. 3 shows that due to the adhesive load the relation between the real contact area and the applied load becomes non-linear. Only in a limited range of $L>0$, the relation between $F_{\mathrm{f}}$ and $L$ can be approximated by a linear equation:

$$
F_{f}=\mu_{e, a}\left(F_{a}^{0}+L\right)
$$

In which the influence of the adhesive load is accounted for by the (apparent) zero load adhesion force $F_{\mathrm{a}}{ }^{0}$ and the friction coefficient $\mu_{\mathrm{e}, \mathrm{a}}=\mathrm{d} F_{\mathrm{f}} / \mathrm{d} L$ for elastic adhesive contact. The friction coefficient accounts for the increase of the adhesive load with increasing $L$, which is caused by the fact that the number of contacting asperities increases with increasing applied load.

\subsection{Elastic Limits}

The model that is described so far is based on elastic deformation in the contact points. Plastic deformation becomes important when the maximum of the Hertzian pressure approaches $H$, with $H=H_{1} / / H_{2}$ the hardness of the contacting materials [17]. The maximum pressure $q_{0}$ in the Hertzian contact is given by: 


$$
z=\frac{4}{9} \pi^{2} q_{0}^{2} D^{2} R
$$

Where $z$ is the indentation. For the Gaussian distribution model, the conditions for plastic or elastic contact can be represented in a dimensionless group, the plasticity index [17]. Significant plastic deformation develops if the mean real elastic contact pressure as defined in eq. (7) is larger than a certain fraction of the hardness: $p_{\mathrm{e}}>0.24 \mathrm{H}$. This condition applies for both adhesive and non-adhesive contacts. As $p_{\mathrm{e}}$ rises beyond $0.24 \mathrm{H}$, with increasing $\sqrt{\sigma / R}$, the mean real contact pressure approaches $H$. For the friction coefficient this means that for $p_{\mathrm{e}}<0.24 H$ the friction coefficient equals $s / p_{\mathrm{e}}$, which is at least four times larger than the lower limit $\mu_{\mathrm{p}}$ $=s / \mathrm{H}$. For $p_{\mathrm{e}}>0.24 \mathrm{H}$ the friction coefficient tends to this lower limit.

\section{FEW CONTACT POINTS}

For small loads the statistical models can predict a number of contact points smaller than one. This results in an underestimate of the adhesive load. The number of contact points has to be at least 1,2 , or 3 depending on the degrees of freedom of the contacting surfaces. Adapting eq. (4a,b) for $N$ equally loaded contact points and application of eq. (1) yields:

$$
\begin{gathered}
F_{f}(L)=s \cdot A_{r}(L)=s \cdot N \cdot \pi R \cdot\left[\frac{1}{N} \frac{D}{R^{1 / 2}} \cdot(L+N \cdot 2 \pi \cdot \Delta \gamma \cdot R)\right]^{2 / 3} \\
(N=1,2,3)
\end{gathered}
$$

\section{MEMS FRICTION EXPERIMENT}

In order to test the clamps of a polysilicon stepper motor [22], a friction meter, similar to [37], has been fabricated. The friction as a function of the applied load has been measured. We have analyzed this experiment using the presented friction theory.

Friction is measured by pushing the shoe against the wall with a defined electrostatic force, and measuring the electrostatic pull force at the onset of slip (fig. 4). The contact material was polysilicon, probably covered with a thin layer of native oxide. Gap-closing actuator arrays have been used, because the moment of slip can easily be determined, as these actuators show pull-in. The measurements have been done using a clamp actuator with 30 plates, and a pull actuator with 15 plates, each plate having an active area of $100 \times 5 \mu \mathrm{m}^{2}$. The total load consists of the clamp force $L$ plus the interfacial adhesive load. The measurements have been carried out under the condition $L>0$, as the clamp voltage is chosen above the level that is necessary to make initial contact. Fig. 5 shows the measured pullvoltage squared as a function of the applied clamp voltages squared. Pull and clamp voltages have been chosen large enough to obtain pull-in of the pull actuator, directly from the initial position. The measurements indicate that there is a close to linear relation between the load $L$ and the $F_{\mathrm{f}}$, and therefore eq. (10) can be used. The clamp force $L$ consists of the generated electrostatic clamp force minus the elastic deformation force of the clamp actuator suspension and the drive beam connected to the pull actuator:

$L=F_{\text {elec }}-F_{\text {spring. }}$. The elastic force $F_{\text {spring }}$ equals $7 \pm 2$ $\mu \mathrm{N}$, calculated from the stiffness of the suspension and the pull connection beam, times the initial gap between the shoe and the rigid wall.

a)

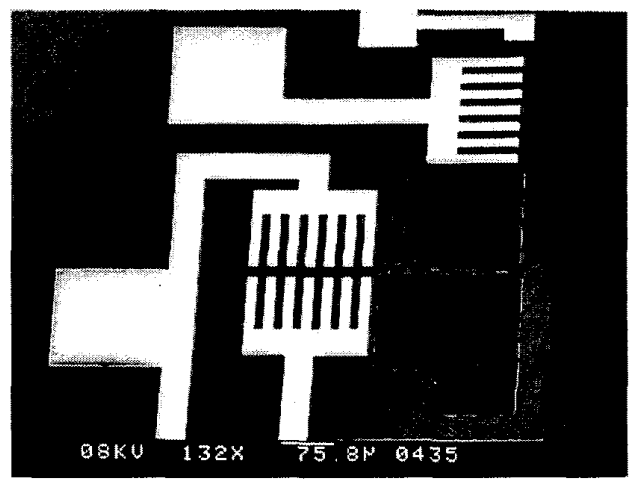

b)

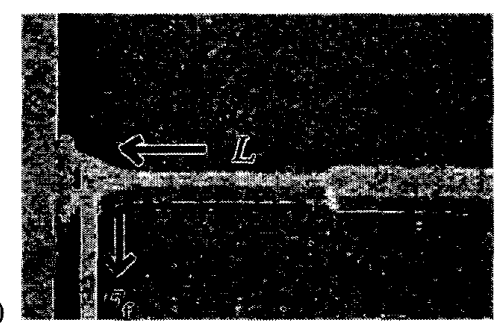

Figure 4a) The friction meter. It consists of an actuator array that lamps a shoe against a rigid wall, and an actuator array pulling the shoe along the wall. Friction is determined by measuring the pull force at the onset of slip of the shoe along the wall. The stator poles of the pull and clamp actuator are white due to charging in the SEM. b) Close-up of the clamp shoe pushed against the rigid side-wall. The height of the shoe is $5 \mu \mathrm{m}$, the width is $20 \mu \mathrm{m}$.

The friction force and the applied load can be obtained by $F_{\text {pull }}=\alpha_{\text {pull }} \cdot V_{\text {pull }}^{2}$ and $F_{\text {elec }}=\alpha_{\text {clamp }} \cdot V_{\text {clamp }}^{2}$, where $\alpha_{\text {pull }}$ and $\alpha_{\text {clamp }}$ are the conversion factors from actuator voltage to force $\left[\mathrm{N} / \mathrm{V}^{2}\right]$ depending on the actuator geometry. Due to uncertainties in the gap sizes of the actuator there is a significant uncertainty in the conversion factors: $\alpha_{\text {clamp }}=(1.4 \pm 0.5) \times 10^{-8} \mathrm{~N} / \mathrm{V}^{2}$ and $\alpha_{\text {pull }}=(3.8 \pm 0.8) \times 10^{-9} \mathrm{~N} / \mathrm{V}^{2}$. Using the conversion factors and subtraction of $F_{\text {spring }}$, the voltages have been converted into forces. Represented in the form of eq. (10) it follows for the measured relation that $\mu_{\mathrm{e}, \mathrm{a}}=0.7 \pm$ 0.3 and $F_{\mathrm{a}}{ }^{0}=F_{\mathrm{f}}(L=0) / \mu_{\mathrm{e}, \mathrm{a}}=2.0 \pm 1.3 \mu \mathrm{N}$. Next, have tried to calculate the friction vs. load using the elastic adhesive contact model. Surface topography parameters have been derived from a top view of a side-wall in the 
friction tester (fig.6). One should realize that the sidewalls are not nominally flat, and that top view SEM photo from which the roughness has been measured may not be completely representative for the whole side-wall. Also possible small asperities (lateral size $<0.1 \mu \mathrm{m}$ ) on top of the larger asperities have been cancelled due to the limited resolution of the roughness measurement. Therefore, the model calculations for this experiment should be considered as an estimate.

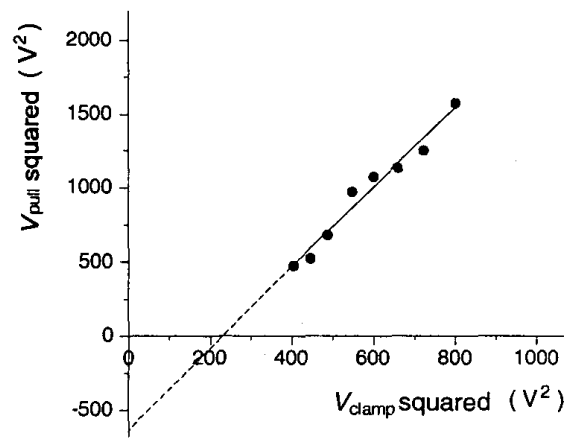

Figure 5: The measured pull voltages squared at the onset of slip, as a function of the applied clamp voltage squared. The measurements have been carried out at a relative humidity of the air of $35 \pm 5 \%$.

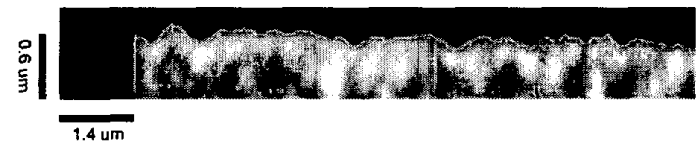

Figure 6: SEM photograph (top view) of the roughness of a side-wall in the friction meter.

\begin{tabular}{|l|l|l|}
\hline Asperity Density & $\eta$ & $4 \times 10^{12} \mathrm{~m}^{-2}$ \\
\hline Asperity Radius & $R$ & $0.5 \pm 0.3 \mu \mathrm{m}$ \\
\hline Work of Adhesion & $\Delta \gamma$ & $0.1 \mathrm{~J} \cdot \mathrm{m}^{-2}$ \\
\hline SD Summit Level & $\sigma$ & $0.04 \mu \mathrm{m}$ \\
\hline Compliance & $D$ & $9.1 \times 10^{-12} \mathrm{~Pa}^{-1}$ \\
\hline Apparent contact area & $A_{\mathrm{a}}$ & $100 \mu \mathrm{m}^{2}$ \\
\hline
\end{tabular}

Table 1: Parameters of the contacting surfaces. The radius $R$, the summit level standard deviation $\sigma$ and the compliance $D$ account for both surfaces. The value of $\Delta \gamma$ is known from wafer bonding with hydrophilic surfaces [38]

The measured parameters (table 1) yield an adhesion parameter $\theta=1 \times 10^{4}$. Using eq. $(5 \mathrm{a}, 6,8)$ the zero load number of contact points can be calculated. It is negligible small, which indicates that the statistical model is not valid, and the discrete contact model has to be used. In order to fix the rotational degrees of freedom, three contact points are needed. Assuming $N=$ 3 in eq. (12), a good correspondence between measured friction $(L>0)$ and calculated friction vs. load is found. The shear strength was used as (the only) fit parameter, in order to fit the calculated slope in $L=3 \mu \mathrm{N}$ with the measured $\mu_{\mathrm{e}, \mathrm{a}}=0.7$. This leads to a value $s=1.4 \mathrm{GPa}$.
With this value an apparent zero load adhesion force $F_{\mathrm{a}}{ }^{0}=s \cdot A_{\mathrm{r}}(L=0) / \mu_{\mathrm{e}, \mathrm{a}}=2.3 \mu \mathrm{N}$ is found (fig. 7). The calculated $F_{\mathrm{a}}{ }^{0}$ is in good correspondence with the measured value. The strong non-linearity of the curve for $L<0$ explains why the apparent zero load adhesion force $F_{\mathrm{a}}{ }^{0}$ is larger than the real calculated adhesion force $F_{\mathrm{a}}=6 \pi \cdot \Delta \gamma \cdot R=0.9 \mu \mathrm{N}$ : For spherical asperities and small total loads $\left(L+F_{\mathrm{a}}\right), \mathrm{d} A_{\mathrm{r}} / \mathrm{d} L$ is large and the friction force rises rapidly with increasing $L$, with a slope larger than $\mu_{\varepsilon, \mathrm{a}}$. With the help of eq. (11) and assuming a hardness of $9 \mathrm{GPa}$ for polysilicon, it was confirmed that the deformations in the contact are indeed elastic.

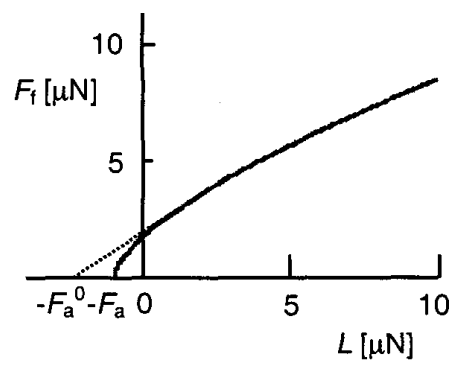

Figure 7: $F_{f}$ as a function of $L$ in the discrete model with 3 contact points, for $R=0.5 \mu \mathrm{m}$ and $s=1.4 \mathrm{GPa}$. The shear strength has been fit to obtain $\mu_{\mathrm{e}, \mathrm{a}}=0.7$ for $L>0$. The relation between $F_{\mathrm{f}}$ and $L$ yields a good fit with the measured $F_{\mathrm{a}}{ }^{0}=$ $2 \pm 1.3 \mu \mathrm{N}$.

\section{CONCLUSIONS}

According to the presented friction models the following conclusions can de drawn for shearing mode static friction: For zero adhesive load, the friction force as a function of the load is given by Amontons' Law. For plastic contact the friction coefficient is given by $s / H$ and for elastic contact by $s / p_{\mathrm{c}}$. For elastic contact, the mean real contact pressure depends on the surface topography: It increases proportional to $\sigma / R$ until the $p_{\mathrm{e}}$ is so large that plastic deformation starts. The friction coefficient decreases with increasing $\sigma / R$. The lower limit is given by the friction coefficient for plastic contact $\mathrm{s} / \mathrm{H}$. The adhesive load is proportional to the number of contacting asperities, and therefore it depends on the applied (external) load. In the statistical model the influence of adhesion can be accounted for by a single dimensionless number, the adhesion parameter. For low loading conditions the statistical models can predict an unrealistic low number of contact points and underestimate the adhesive load. In these cases 1,2 or 3 should be taken for the number of contact points, depending on the degrees of freedom of the contacting members. A MEMS friction experiment has been analyzed in order to relate the measured friction with surface topography, adhesion energy and elastic properties. The experiment illustrates the need for special attention to low loading conditions, which can be 
encountered in MEMS. Future work should concentrate on experimental verification of the presented models. How to relate the idealized roughness representation to the real roughness will be a primary issue.

\section{ACKNOWLEDGEMENT}

The authors wish to thank Bart Vogelzang for his contribution to the analysis of friction in adhesive contacts, and Tonny Sonnenberg for his contribution to the friction measurements.

\section{REFERENCES}

1. Tai Y-C, Muller R.S.,"Frictional study of IC-processed motors", Sensors and Actuators, A21-23, 1990,.180-183.

2. Mehregany M., Nagarkar P., Senturia S.D., Lang J.H.,"Operation of microfabricated harmonic and ordinary side-drive motors", Proc. IEEE Workshop on MEMS, Napa Valley, CA, Feb. 1990 pp. 1.8 .

3. Lim M.G., Chang J.C., Schultz D.P., Howe R.T., White R.M.,"Polysilicon microstructures to characterize static friction", Proc. IEEE Workshop on MEMS, Napa Valley, CA, Feb. 1990, pp. 82-88.

4. Kaneko R.,"Microtribology related to MEMS", Proc. IEEE Workshop on MEMS, Nara, Japan, Jan. 30 - Feb.2 1991, pp. 1-8.

5. Yeh R., Kruglick E.J.J., Pister K.S.J.,"Microelectromechanica components for articulated microrobots", The $8^{\text {th }}$ Int. Conf. on Solid-State Sensors and Actuators, and Eurosensors IX Stockholm, Sweden, June 25-29, 1995, pp. 346-349.

6. Gabriel K.J., Behi F., Mahadevan R.,"In situ friction and wear measurenemts in integrated polysilicon mechanisms",Sensors and Actuators, A21-23, 1990, pp. 184-188.

7. Mehregany M.,"Friction and wear in microfabricated harmonic side-drive motors", Techn. Digest of the IEEE Solid-state Sensors and Actuators Workshop, Hilton Head Island, South Carolina, June 1990, pp. 17-22.

8. Suzuki S., Matsuura, Uchizawa M., Yura S., Shibata H.,"Friction and wear studies on lubricants and materials applicable to MEMS", Proc. IEEE Workshop on MEMS, Nara, Japan, Jan. 30 Feb. 2 1991, pp. 143-147.

9. Noguchi K., Fujita H., Suzuki M.,"'The measurement of friction on micromechatronic elements", Proc. IEEE Workshop on MEMS, Nara, Japan, Jan. 30 - Feb.2 1991, pp. 148-153.

10. Mehregany M., Tai Y-C,'Surface micromachined mechanisms and micromotors", J. Micromech. Microeng., vol. 1, 1991, pp. 73-85.

11. Deng K., Ko W.H.,"A study of static friction between silicon and silicon compounds", J. Micromech. Microeng., vol.2, 1992 pp.14-20.

12. Mehregany M., Senturia S.D., Lang J.L., Nagarkar P.,"Micromotor fabrication", IEEE Trans. on Electron Dev., vol. 39, no. 9, Sept. 1992 , pp. 2060-2069.

13. Huang J.-B., Mao P.-S, Tong Q.-Y. and R.-Q. Zhang,"Study on Si electrostatic electroquasistatic micromotors", Sensors and Actuators A, vol. 35, 1993, pp. 171-174

14. Garcia E.J., Sniegowski J.J.,"Surface micromachined microengine", Sensors and Actuators $A$,Vol. 48, 1995, pp. 203214.

15. Beerschwinger U., Reuben R.L., Yang S.J.,"Frictional study of micromotor bearings", Sensors and Actuators A, vol. 63, 1997 pp. 229-241.

16. Komvopoulos K.,"Surface engineering and microtribology for microelectromechanical systems", Wear, Vol. 200, 1996, pp 305-327.
17. Greenwood J.A., Williamson J.B.P.,"Contact of nominally flat surfaces", Proc. R. Soc. Lond. A, Vol. 295, 1966, pp. 300-319.

18. Maugis D.,"On the contact and adhesion of rough surfaces", J. Adhesion Sci. Technol, vol. 10, no.2, 1996, pp. 161-175.

19. Majumdar A., Bhushan B., in:"Handbook of micro-and nano tribology" ed. Bhushan B., CRC Press, New York, 1995.

20. Alley R.L., Mai P., Komvopoulos, Howe R.T.,"Surface roughness modifications of interfacial contacts in polysilicon microstructures", Proc. $7^{\text {th }}$ Int. Conf. on Solid-State Sensors and Actuators, Yokohama,Japan, June 7-10, 1993, 288-291.

21. Gui C., Elwenspoek M., Tas N., Gardeniers J.G.E.,"The effect of surface roughness on direct wafer bonding", J. Appl. Phys., Vol. 85 , issue 10,1999 , pp. 7448-7454.

22. Tas N.R., Sonnenberg A.H., Sander A.F.M. and Elwenspoek M.,"Surface micromachined linear electrostatic stepper motor", Proc. IEEE Workshop on MEMS, Nagoya, Japan, Januari 26-30, 1997, 215-220.

23. Bowden F.P., Tabor D.,"The friction and lubrication of solids", Oxford Clarendon Press, 1950.

24. Tabor D.,"Friction-The present state of our understanding", J. Lubrication Techn., vol. 103, 1981, pp.169-179.

25. Clechet P., Martelet C., Belin M., Zarrad H., Jaffrezic-Renault N., Fayeulle S.,"Lubrication of silicon micromechanisms by chemical grafting of long-chain molecules", Sensors and Actuators $A$, vol. 44,1994 , pp. 77-81.

26. Zarrad H., Chovelon J.M., Clechet P., Jaffrezic-Renault N., Martelet C., Belin M., Perez H., Chevalier Y.,"Optimization of lubricants for silica micromotors", Sensors and Actuators $A$, vol. 46-47, 1995, pp. 598-600.

27. Deng, K., Collins R.J., Mehregany M., Sukenik C.N.,"Performance impact of monolayer coating of polysilicon micromotors", J. Electrochem. Soc., vol. 142, no.4, April 1995 pp. 1278-1285.

28. Bhushan B.,"Nanotribology and nanomechanics of mems devices",Proc. IEEE Workshop on MEMS, SanDiego, CA, Feb. 14-15, pp. 349-353.

29. Srinivasan U., Foster J.D., Habib U., Howe R.T., Maboudian R. Cowell Senft D., Dugger M.T.,"Lubrication of polysilicon micromechanisms with self-assembled monolayers", Techn. Digest of the Solid-State Sensors and Actuator Workshop, Hilton Head Island, South Carolina, Jun. 8-11, 1998, pp. 165-161.

30. Landau L.D., Lifschitz E.M.,'Elastizitaetstheorie", Akademie Verlag, Berlin, 1975.

31. Timoshenko S.P., Goodier J.N.,"Theorie of Elasticity", McGrawHill, New York 1970.

32. Derjaguin B.V., Muller V.M., Toporov Yu. P.,"Effect of contact deformations on the adhesion of particles", J. Colloid Interface Sci., Vol. 53, no. 2, Nov. 1975, pp. 314-326.

33. Johnson K.L., Kendall K., Roberts A.D.,"Surface energy and the contact of elastic solids", Proc. R. Soc. Lond. A, Vol. 324, 1971, pp. 301-313.

34. Tabor D.,"Surface forces and surface interactions", J. Colloid Interface Sci., Vol. 58, no.1, 1977, pp.2-13.

35. Archard,"Surface topography and tribology", Tribology Int., Oct. 1974, pp. 213-220.

36. Fuller K.N.G., Tabor D.,"The effect of surface roughness on the adhesion of elastic solids", Proc. R. Soc. Lond. A., vol. 345, 1975, pp.327-342

37. Prasad R., MacDonald, N., Taylor D.,"Micro-instrumentation for tribological measurement", $8^{\text {th }}$ Int. Conf. Solid-State Sensors and Actuators, Transducers' 95 and Eurosensors IX, Stockholm Sweden, June 25-29, 1995

38. Stengl R., Tan T., Gosele U.,"A model for the silicon wafer bonding process", Jap. J. of App. Phys., Vol. 28, no. 10, 1989 pp. 1735-1741. 